\title{
Flexible Hilbert-Curve Loop Antenna Having a Triple-Band and Omnidirectional Pattern for WLAN/WiMAX Applications
}

\author{
Dang-Oh Kim, Che-Young Kim, and Dae-Geun Yang \\ School of Electronics Engineering, Kyungpook National University, Sankyuk-dong Puk-gu, Daegu 702-701, Republic of Korea \\ Correspondence should be addressed to Che-Young Kim, cykim@ee.knu.ac.kr
}

Received 14 December 2011; Revised 20 January 2012; Accepted 25 January 2012

Academic Editor: Ana Collado

Copyright () 2012 Dang-Oh Kim et al. This is an open access article distributed under the Creative Commons Attribution License, which permits unrestricted use, distribution, and reproduction in any medium, provided the original work is properly cited.

A triple-band flexible loop antenna is proposed for WLAN/WiMAX applications in this paper. The proposed antenna is formed by the third-order Hilbert-curve and bending type structure which provides flexible characteristics. Even though the radius of the curvature for bending antennas is changed, a triple-band feature still remains in the proposed antenna. Moreover, the antenna exhibits the characteristics of omnidirectional radiation pattern and circular polarization. To verify the receiving performance of antenna, a simulation on the antenna factor was conducted by an EM simulator. Based on these results, the suggested antenna makes a noteworthy performance over typical loop antennas.

\section{Introduction}

Recently, the proliferation of radio devices in diverse shapes and rapid progress in flexible technology require the antennas mounted on wireless devices to be manufactured in various shapes. Furthermore, vibrant studies of wearable systems, RFID tags, and flexible displays have spurred on much attention to the flexible antenna technology. Especially, there are increasing requirements of flexible antennas with lowprofile, compact size, and multiband including the wireless local area network (WLAN) band $2.4 / 5.2 / 5.8 \mathrm{GHz}$ and the world interoperability for microwave access (WiMAX) band $3.5 \mathrm{GHz}$ due to the explosive growth of demand for wireless data communication. The antennas operating for wearable systems and RFID tags are prone to the proximity effect by the material itself so that they usually suffer from degrading performance due to the resonating currents on the ground plane and their compact size. Particularly, these weaknesses mainly occur in the antennas used for data communications. Once encountered, to mitigate the performance degradation, a loop antenna has been an alternative since the current induced on the ground surface becomes small in magnitude [1-3]. Consequently, the loop antenna is regarded as a reference antenna in this paper.

This paper presents a flexible Hilbert curve loop antenna endorsing the Hilbert curve over the typical rectangular loop, which is aimed for WLAN/WiMAX applications. The suggested antenna possesses the flexible bending structure and shows triple-band characteristics at $2.4 / 3.5 / 5.5 \mathrm{GHz}$ operating bands. In addition, the antenna exhibits the omnidirectional pattern without null in comparison to a traditional loop antenna. In order to check the receiving performance of antenna, the antenna factor $K$ is examined with an EM simulator. The structure of our proposed antenna is different from conventional flexible antennas such as spiral curve-shaped monopole antenna [4], conventional patchshaped antenna [5], meander line antenna $[6,7]$, combshaped monopole antenna [8], inverted-F-shaped antenna [9], ultra-wideband (UWB) antenna [10], the zeroth-order resonant (ZOR) antenna [11-13], coplanar waveguide(CPW-) fed monopole antenna [14], and folded slot dipole antenna [15]. Moreover, this is the first time the Hilbert-curve geometry to a loop antenna has been applied to such an application.

\section{Description of the Antenna}

In this study, the Hilbert curve, first proposed by Hilbert in 1891, was used as the space-filling curve structure. The Hilbert-curve geometry in antenna design has been explored to reduce the antenna size and to make it operate at multiple 


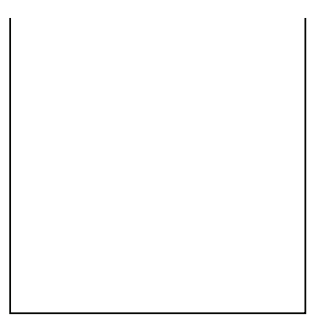

(a)

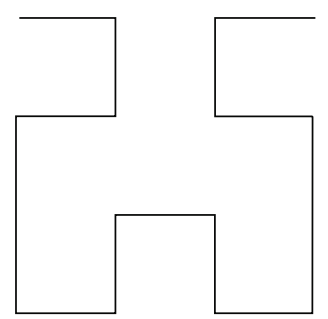

(b)

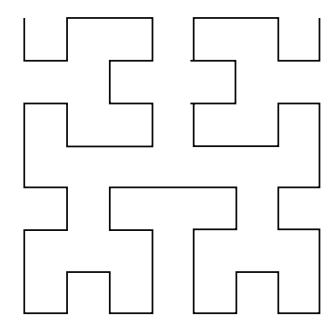

(c)

Figure 1: The Hilbert curves with increasing iteration order number $n$. (a) First order, (b) Second order, and (c) Third order.

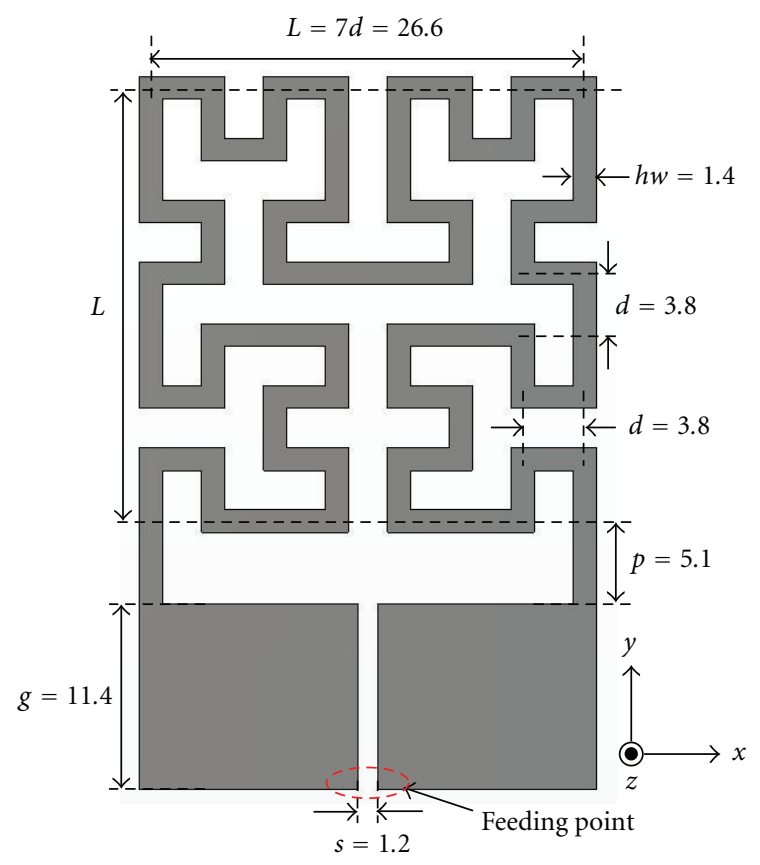

FIGURE 2: Geometry and dimensions of the proposed Hilbert-curve loop antenna.

frequencies [16-22]. In addition, recent research has shown the use of this curve in the designs of the circular polarized antennas, metamaterial resonators, and high impedance frequency-selective structures (FSSs) [23-26]. The issue on the Hilbert-curve geometry is to find the most effective way to fill the lines in the fixed space. The Hilbert antenna has shown to be an effective structure to obtain small antennas [27]. The performance of the meander line is similar to the Hilbert curve. But unlike the meander line, the Hilbertcurve takes the advantage of tagging the iteration order number associated with geometries and providing other performances such as circular polarization. Figure 1 shows the first few iterations order of Hilbert curve geometry. It is self-evident that as the iteration order increases, the total length of the line segment is increased thereafter. The sum of line segment $S$ is given by $[18,24]$

$$
\begin{gathered}
S=\left(2^{2 n}-1\right) d=\left(2^{n}+1\right) L, \\
d=\frac{L}{2^{n}-1},
\end{gathered}
$$

where $L$ is the side dimension of the Hilbert curve, $d$ is the length of each line segment, and $n$ indicates the order of iteration. Namely, as the iteration order is increased, the total length of the line segment is increased while its footprint size is kept fixed. From the electrical resonance length point of view, this property is what allows the Hilbert curve to possess a relatively low resonant frequency, which could lead to the miniaturization of structure. In this paper, the Hilbert curve with the third-order $(n=3)$ iteration was fit into a loop antenna forming a closed-loop configuration. Figure 2 shows the configuration and dimensions of the flat Hilbert curve loop antenna in a flat shape without bending. The proposed antenna consists of only a copper film without the dielectric substrate. The top part of the antenna is the third-order Hilbert curve where the bottom part of antenna reaches to the feeding point as shown in Figure 1(c). The matching plane to tune the input impedance of the antenna is embedded in the feeding part. With adjustment of the length $g$ and $s$ sizing the matching plane, the impedance matching is accomplished. Also, the aggregated structure 


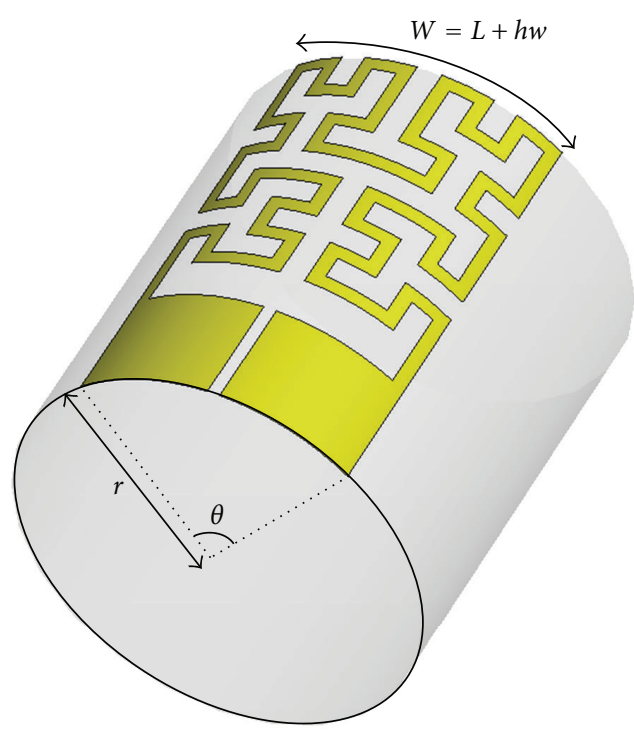

Figure 3: Geometry of the flexible Hilbert-curve loop antenna and radius of its curvature.

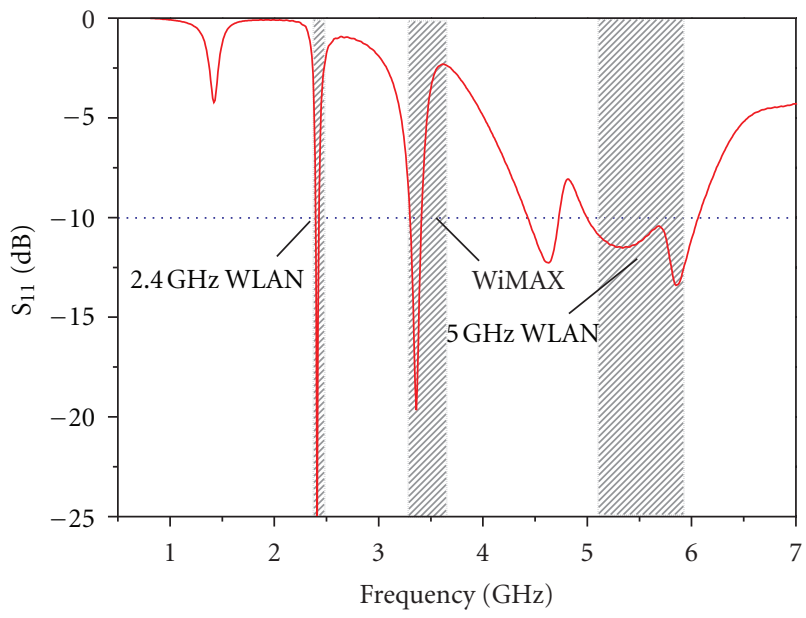

Figure 4: $S_{11}$ results of the flexible Hilbert-curve loop antenna.

maintains its symmetry about the center line. All of the geometrical parameters of the antenna were optimized by Microwave Studio of CST. Figure 3 shows the radius of curvature of the bending structure and the geometry bent to the flexible Hilbert-curve loop antenna as shown in Figure 2. The antenna of the bending structure with the fixed width $W$ has the radius of curvature $r$ with a corresponding bending level. The degree of bending is measured by the radius of curvature by

$$
r=\frac{180 \cdot W}{\pi \theta}
$$

\section{Results and Discussion}

Figure 4 shows the simulated $S_{11}$ plots of the finalized flexible Hilbert-curve loop antenna with $55 \mathrm{~mm}$ radius of

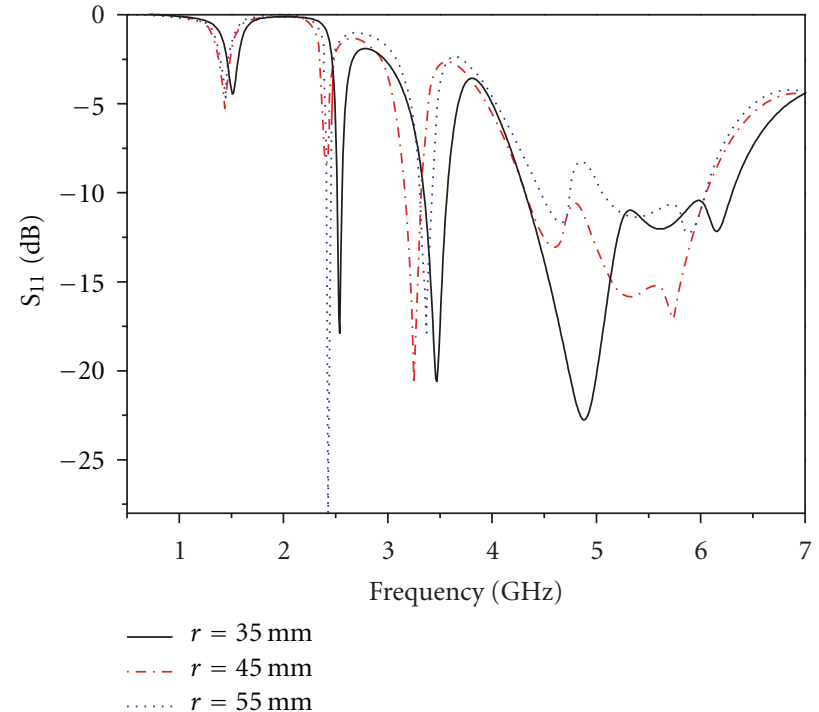

FIGURE 5: $S_{11}$ results of the proposed antenna in terms of the radius of curvature.

curvature. As depicted by the simulation result, this loop antenna operates at a frequency of WLAN band $2.45 \mathrm{GHz}$, WiMAX band $3.35 \mathrm{GHz}$. Moreover, the antenna covers the entire $5 \mathrm{GHz}$ WLAN bands. In here, the side dimension $L$ of the Hilbert curve can be put to about $0.2 \lambda$ (at $2.45 \mathrm{GHz}$ ), $0.3 \lambda$ (at $3.45 \mathrm{GHz}$ ), and $0.5 \lambda$ (at $5.5 \mathrm{GHz}$ ). The simulated $S_{11}$ plots of the antennas bent with different radii of curvature are shown in Figure 5. This figure reveals that the return loss result of the antenna is changed by the adjustment of the bending level of the proposed antenna. Although the resonant frequencies were marginally shifted to adjacent frequency owing to antenna bending, the operating frequencies of the antenna were still maintained in the targeted band within the limit of the partial radius of curvature. From this result, the proposed antenna can be adaptable to bending structures and finds its way to flexible applications.

Figure 6 shows the simulated omnidirectional radiation pattern of the proposed flexible Hilbert-curve loop antenna. The radiation pattern in Figure 6 is suitable for the conventional WLAN/WiMAX communication system requiring the omnidirectional pattern. In addition, its most notable distinction of radiation pattern is a uniform pattern without nulling. The maximum gains and radiation efficiencies of antenna are $3.96 \mathrm{dBi}(0.88)$ at $2.45 \mathrm{GHz}, 2.88 \mathrm{dBi}(0.96)$ at $3.35 \mathrm{GHz}$, and $3.84 \mathrm{dBi}(0.92)$ at $5.5 \mathrm{GHz}$ (parenthesis designates the radiation efficiency). Figure 7 shows the simulated surface current distributions at each resonance frequency. The applied power to each antenna is 1 Watt. The large current distribution is indicated in dark color, and the small one is in light color. It is seen that the resonant surface currents concentrated on the Hilbert curve at $2.4 \mathrm{GHz}$ and $3.5 \mathrm{GHz}$ are higher than the case of $5.5 \mathrm{GHz}$. This means that many segments of the Hilbert curve at $5.5 \mathrm{GHz}$ could not contribute effectively to the working role of the individual antenna segment. 

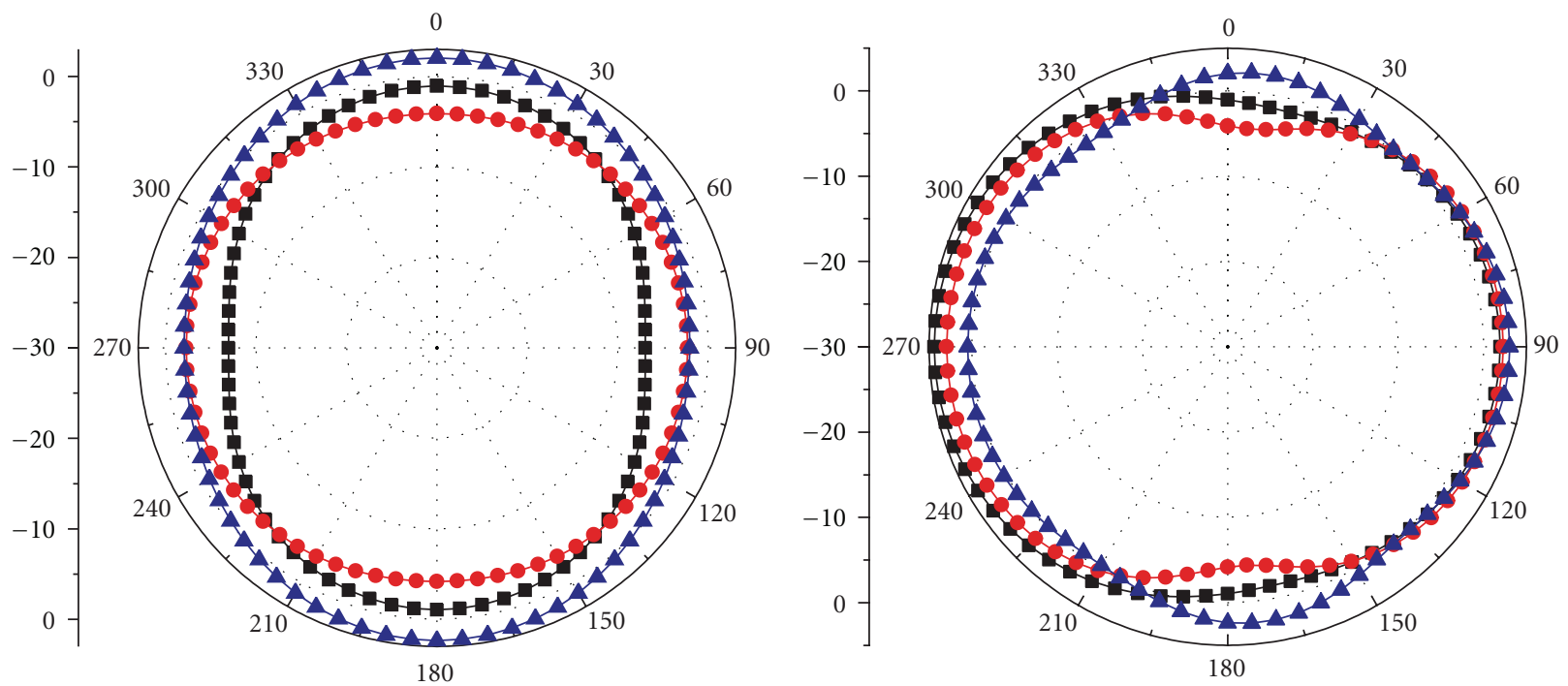

(a)

(b)

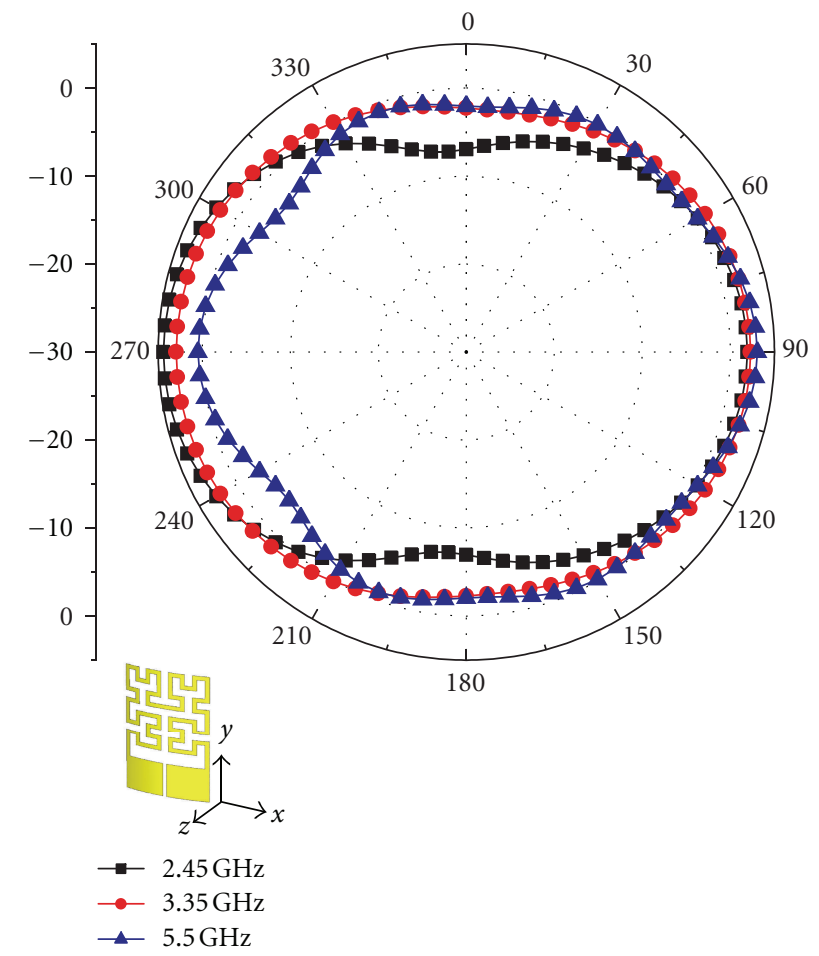

(c)

FIGURE 6: Simulated radiation patterns of the proposed antenna at 2.45/3.35/5.5 GHz. (a) xz-plane, (b) $y z$-plane, and (c) $x y$-plane.

To demonstrate the superiority of the radiation pattern and receiving performance of the proposed antenna, the conventional rectangular loop antennas operating at each triple band were introduced as a reference antenna in this study. This was rooted in the idea that the operation mechanism and characteristics of the rectangular loop antenna are similar to that of classical circular loop antenna; thus, this rectangular antenna could be a representative of the whole loop antenna group. Further, the rectangular loop antenna can be analogized with the primitive structure of the proposed Hilbert-curve loop antenna. The geometry and dimensions of the imposed rectangular loop antennas are shown in Figure 8 and tabulated in Table 1. The feeding structure of this antenna is the same as one of the Hilbert-curve loop antennas. For fair comparison between the flexible Hilbert-curve loop antenna and the rectangular loop antenna, three 


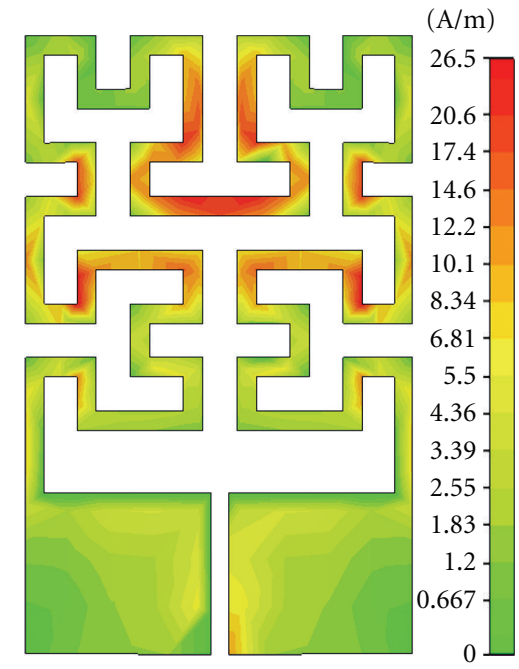

(a)

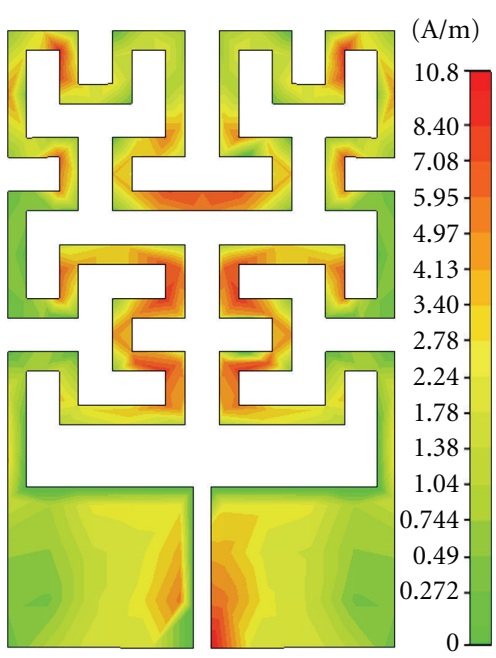

(b)

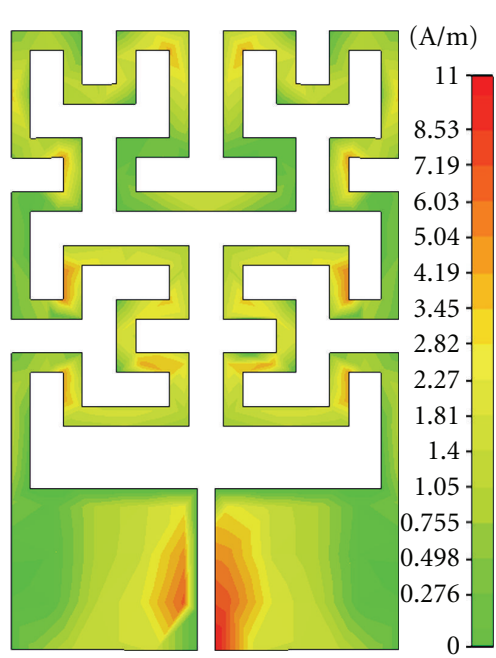

(c)

FIgURE 7: Surface current distribution of the proposed antenna at resonance frequencies. (a) $2.45 \mathrm{GHz}$, (b) $3.35 \mathrm{GHz}$, and (c) $5.5 \mathrm{GHz}$.

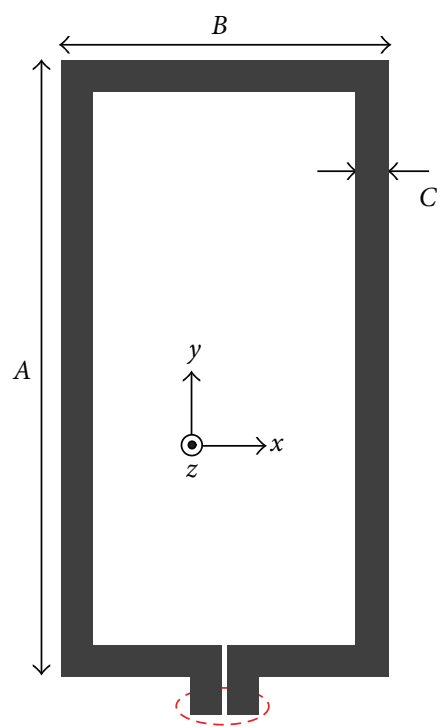

Feeding point

FIgURE 8: Geometry of the reference rectangular loop antenna.

TABLE 1: Dimensions of the reference rectangular loop antenna.

\begin{tabular}{lccc}
\hline Dimension & A & B & C \\
\hline Size $(\mathrm{mm})$ for $2.45 \mathrm{GHz}$ & 48.8 & 26 & 2.5 \\
Size $(\mathrm{mm})$ for $3.35 \mathrm{GHz}$ & 37 & 19.1 & 2.5 \\
Size $(\mathrm{mm})$ for $5.5 \mathrm{GHz}$ & 22 & 11.8 & 1.5 \\
\hline
\end{tabular}

rectangular loop antennas corresponding to each operating band $2.45 / 3.35 / 5.5 \mathrm{GHz}$ were designed since the rectangular loop antenna has generally a single operating frequency. The simulated return loss results of the designed rectangular loop antennas are plotted in Figure 9. Note that the size of the Hilbert-curve loop is only $26.6 \mathrm{~mm} \star 26.6 \mathrm{~mm}$ compared to

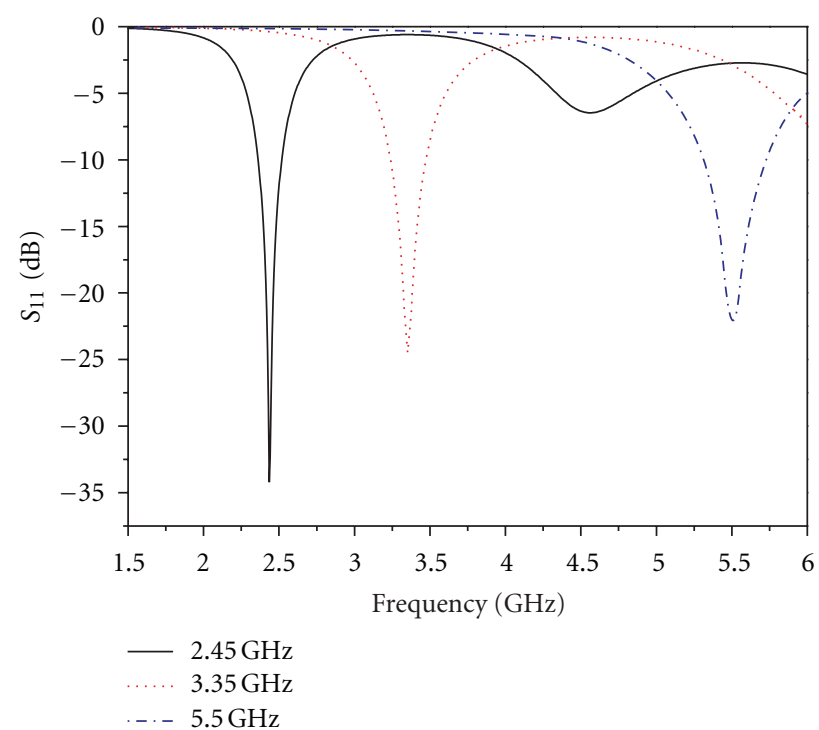

FIGURE 9: $S_{11}$ results of the rectangular loop antennas with each operating band.

$48.8 \mathrm{~mm} \star 26 \mathrm{~mm}$ of the rectangular loop, that is, a reduction of two times the area. Figure 10 shows the radiation pattern of the rectangular loop antenna at $2.45 \mathrm{GHz}$. This pattern is the general radiation pattern of a loop antenna, so other bands such as $3.35 \mathrm{GHz}$ and $5.5 \mathrm{GHz}$ are similar to the pattern of Figure 10. In comparing Figures 6 and 10, in this regard, it is observed that the proposed flexible Hilbert-curve loop antenna achieved an even radiation pattern without null, which indicates that the pattern of the proposed antenna is superior to the pattern of the conventional loop antenna.

To confirm the omnidirectional receiving performance of the proposed antenna, the antenna factor $K$ was used in this 


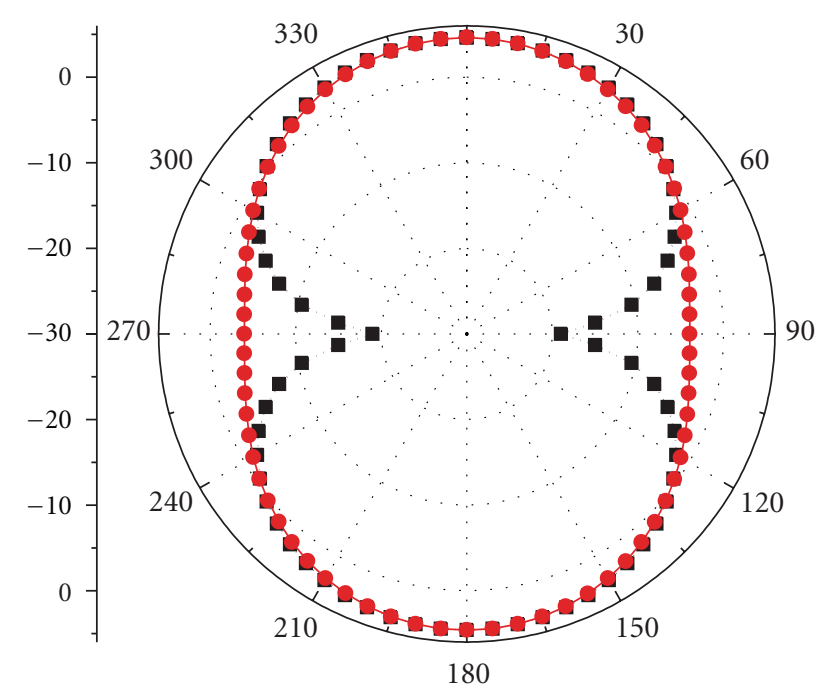

-. $x z$-plane

- $y z$-plane

FIGURE 10: Simulated radiation patterns of the reference rectangular loop antenna at $2.45 \mathrm{GHz}$.

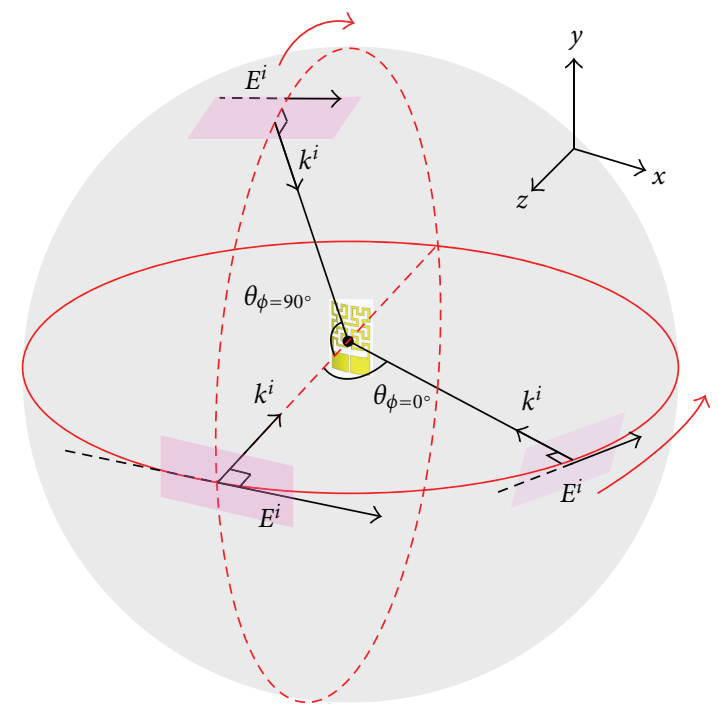

FIGURE 11: Simulation setup of the incident plane wave to obtain the antenna factor $K$.

paper. The antenna factor is an important antenna parameter to determine the receiving sensitivity of a radio communication system, which becomes one of the significant parameters directly affecting the performance of the WLAN/WiMAX communication system. To calculate the antenna factor, a load resistor of $50 \Omega$ was connected to the feeding point of the proposed Hilbert loop antenna and rectangular loop antenna. Then, the induced voltage across a load resistor was developed for the incident plane wave. With the obtained voltage, the antenna factor $K$ was calculated through the following equation [28]:

$$
K=\frac{E^{i}}{V_{A}}\left[\mathrm{~m}^{-1}\right],
$$

where $V_{A}$ is the voltage induced on the loaded loop antenna and $E^{i}$ is the amplitude of the electric field of the incident

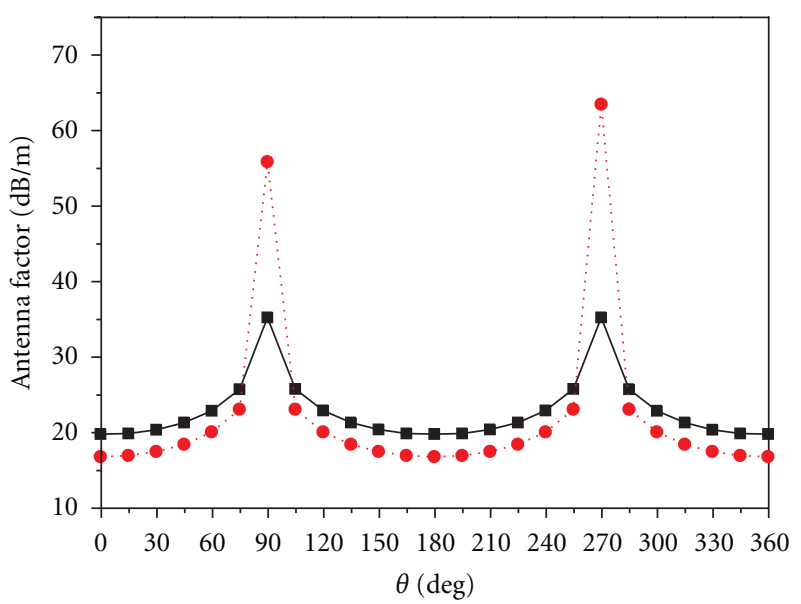

(a)

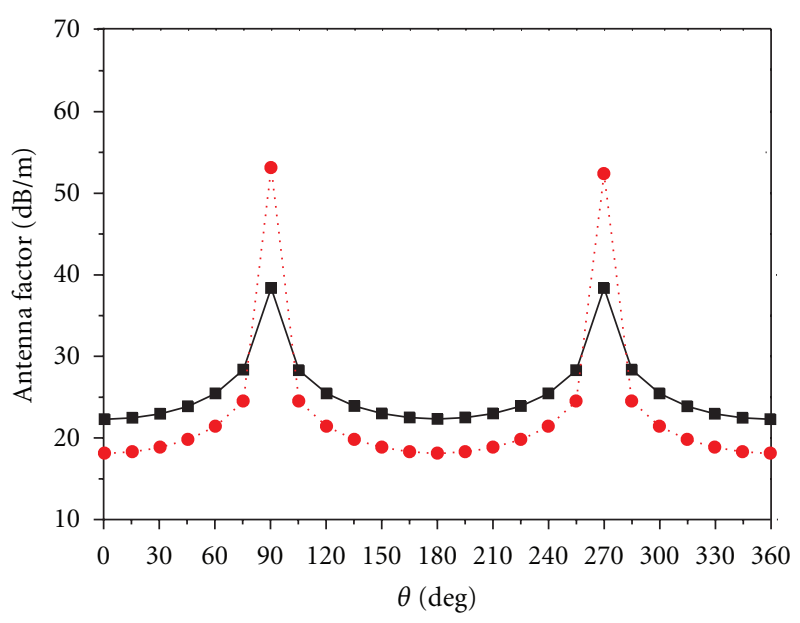

(b)

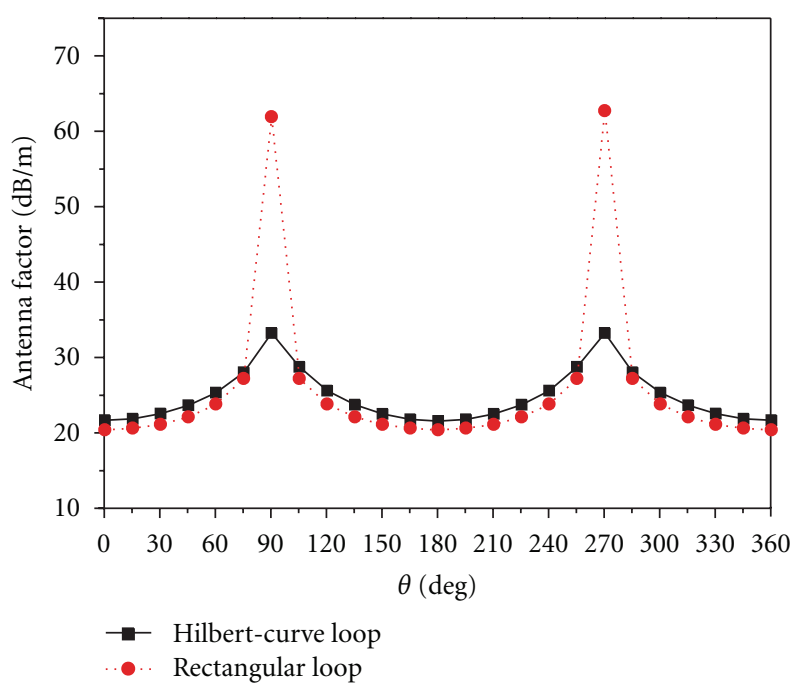

(c)

Figure 12: Variation of the antenna factor $K$ at $\phi=0^{\circ}$ ( $x z$-plane) for the proposed flexible Hilbert-curve loop antenna and rectangular loop antenna. (a) $2.45 \mathrm{GHz}$, (b) $3.35 \mathrm{GHz}$, and (c) $5.5 \mathrm{GHz}$. 
plane wave. Based on the aforementioned simulation, the resultant antenna factor for the various directions of the incident plane wave could be obtained for the proposed antenna and rectangular loop antenna. Figure 11 depicts the simulation configuration of the antenna factor. The amplitude of the incident electric field is the stationary $1[\mathrm{~V} / \mathrm{m}]$, and an incident plane wave is propagating toward the center of the antenna. The incident angle of plane wave has two cases: one with $\phi=0^{\circ}$ ( $x z$-plane) and the other with $\phi=90^{\circ}$ ( $y z$-plane) for all of the rotated angle $\theta$ in this simulation. The polarization of the incident wave kept its direction as shown in Figure 11. The simulation in Figure 11 was performed for the proposed flexible Hilbert-curve loop antenna at each operating band and for the three rectangular loop antennas. The outcomes are indicated in Figures 12 and 13.

The relative variation of the antenna factor is given in Figures 12 and 13 because the proposed and the reference antenna do have their own physical length for respective resonances. As seen in Figure 12, the deviations of the antenna factor of the proposed flexible Hilbert-curve antenna for $\phi=0^{\circ}$ (xz-plane) and all operating bands are within 10 20 $[\mathrm{dB} / \mathrm{m}]$, whereas the rectangular loop antennas are in range of $40 \sim 50[\mathrm{~dB} / \mathrm{m}]$. It was observed that the shape of the curve in Figure 12 was also in good agreement with the radiation pattern of the antenna in Figures 6 and 10. In other words, the peaking angles of the antenna factor observed at $\theta=90^{\circ}$ and $\theta=270^{\circ}$ correspond to that of the null angle appeared in the radiation pattern. Moreover, the antenna factor at $\phi=90^{\circ}$ ( $y z$-plane) of the proposed antenna was $2.8 \sim 3.3[\mathrm{~dB} / \mathrm{m}]$, while the rectangular loop antennas were 4.4 4.7 $[\mathrm{dB} / \mathrm{m}]$ as shown in Figure 13. As seen in Figures 12 and 13, a big difference in the antenna factor between the two antennas was observed at $\phi=0^{\circ}$ whereas a small difference was observed at $\phi=90^{\circ}$. To sum up, the proposed antenna reveals more omnidirectional characteristics than the conventional loop antenna. This feature values the proposed antenna for wireless communication applications. We have addressed the usefulness of the antenna factor together with the radiation pattern to evaluate the receiving performance. However, most papers treating similar topics only adhere to the radiation pattern rarely mentioning the antenna factor.

Figure 14 represents a degree of circular polarization in terms of axial ratio (AR) with respect to the angle $\theta$ at $\phi=0^{\circ}$ for the proposed and the reference antenna. In general, the conventional loop antennas have a linear polarization with a very high value of axial ratio. However, the proposed flexible Hilbert-curve loop antenna reveals a circular polarization performance (AR $<4 \mathrm{~dB}$ ) as shown in Figure 14. This circular polarization property was observed at $2.45 \mathrm{GHz}$ and $3.35 \mathrm{GHz}$ bands since all the segments of the Hilbert cur ve were cooperative in their phases and magnitudes in these bands. This phenomenon agrees with the result in Figure 7. In recent years, there has been some research on generating the circular polarization from the Hilbert-curve antennas $[23,29]$. Though not appeared toward a boresight, the observed circular polarization may find its usefulness in some applications.

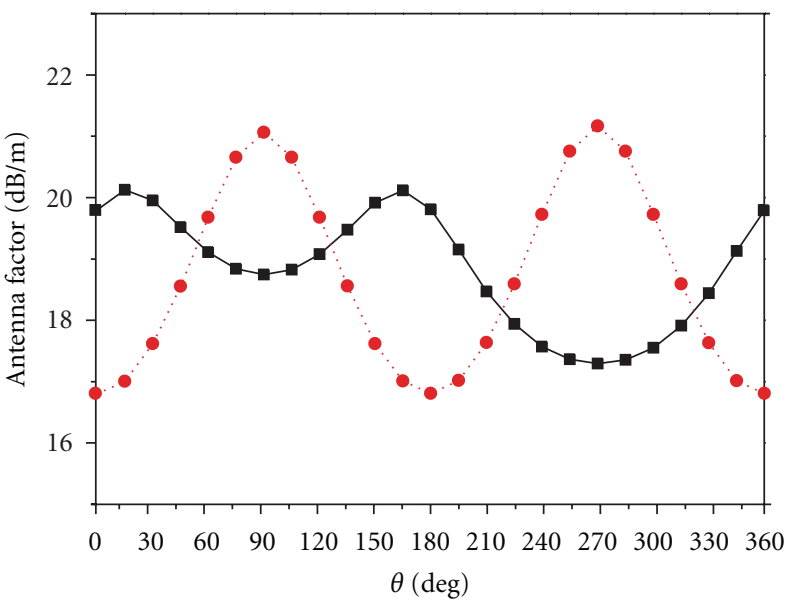

(a)

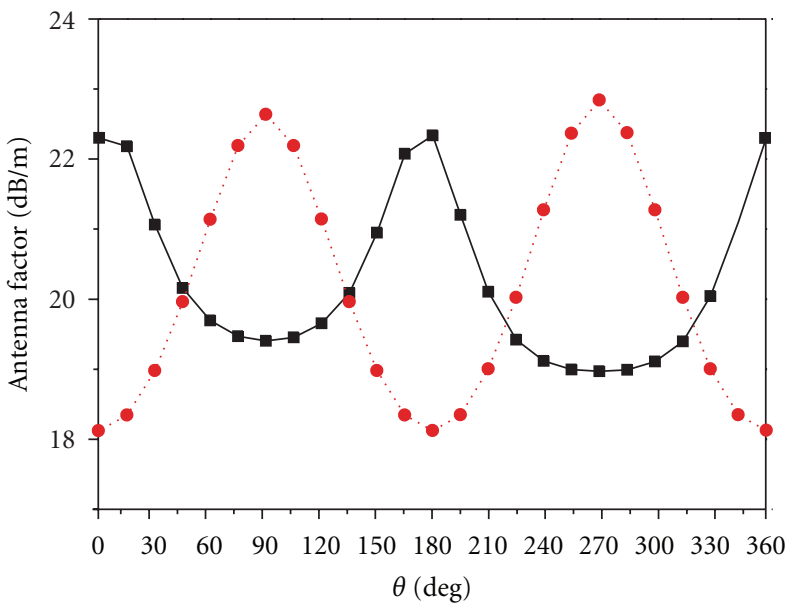

(b)

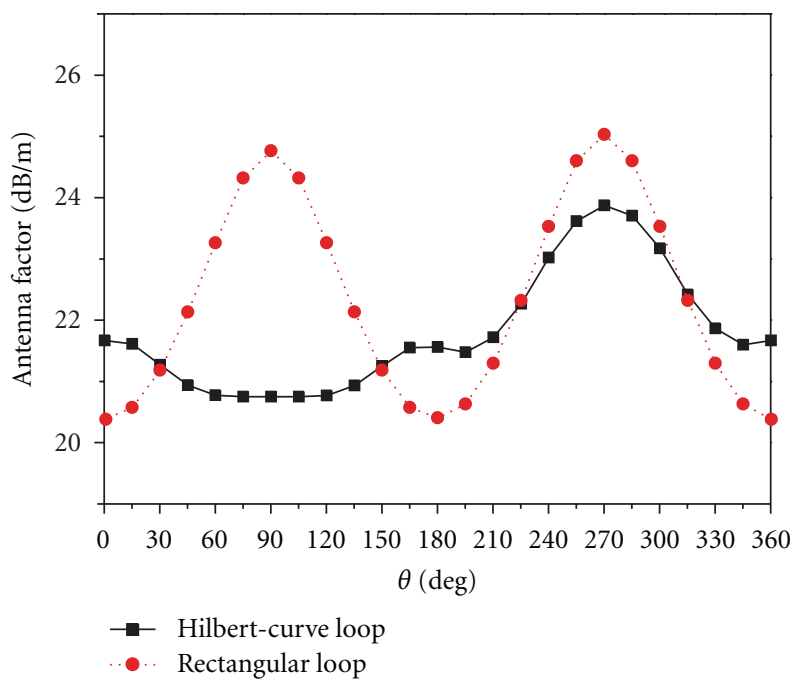

(c)

Figure 13: Variation of the antenna factor $K$ at $\phi=90^{\circ}(y z-$ plane) for the proposed flexible Hilbert-curve loop antenna and rectangular loop antenna. (a) $2.45 \mathrm{GHz}$, (b) $3.35 \mathrm{GHz}$, and (c) $5.5 \mathrm{GHz}$. 


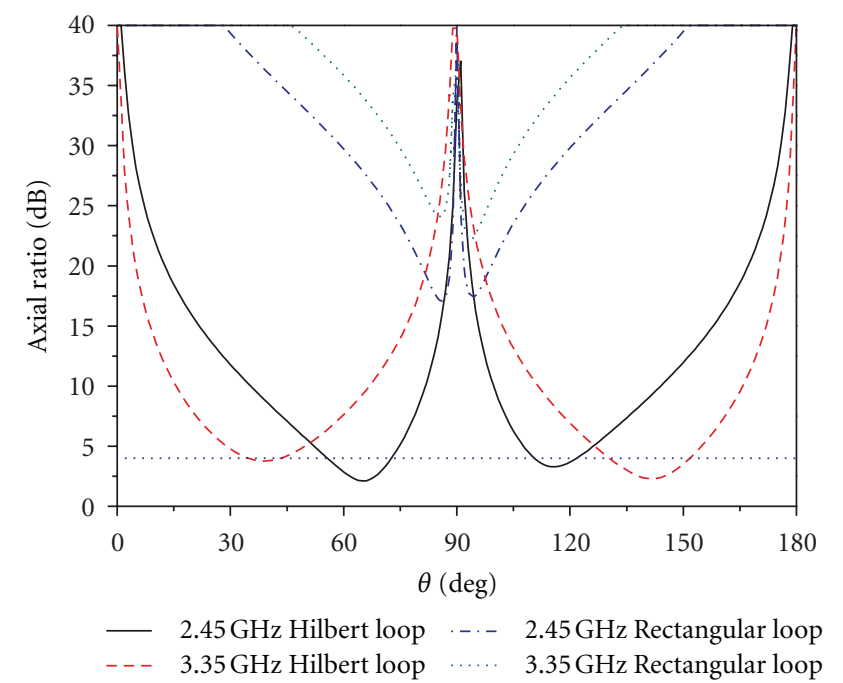

Figure 14: Axial ratio of the proposed flexible Hilbert-curve loop antenna and rectangular loop antenna at $\phi=0^{\circ}$.

\section{Conclusion}

In this paper, the Hilbert-curve loop antenna with an omnidirectional radiation pattern was proposed, and the simulation results are discussed focusing on the flexibility. The finalized antenna possesses the triple-band performance for the WLAN/WiMAX communication system. To compare with the proposed antenna performance, the rectangular loop antenna was used as a reference antenna. On the basis of the far-field characteristics and the result of antenna factor, it was seen that the proposed antenna achieved good omnidirectional radiation pattern and circular polarization performance. The presented results in this paper will be helpful for designing and constructing a flexible Hilbert-curve loop antenna. Furthermore, the proposed antenna could be suitable for the application in WLAN/WiMAX communication systems.

\section{References}

[1] Y. W. Chi and K. L. Wong, "Internal compact dual-band printed loop antenna for mobile phone application," IEEE Transactions on Antennas and Propagation, vol. 55, no. 5, pp. 14571462, 2007.

[2] Z. Xing, L. Wang, C. Wu, J. Li, and M. Zhang, "Characteristics and application of a novel loop antenna to UHF RFID receivers," International Journal of Antennas and Propagation, vol. 2011, Article ID 480717, 7 pages, 2011.

[3] C. W. Chiu, C. H. Chang, and Y. J. Chi, "Multiband folded loop antenna for smart phones," Progress in Electromagnetics Research, vol. 102, pp. 213-226, 2010.

[4] G. Y. Chen and J. S. Sun, "Design of flexible printed antenna," Electronics Letters, vol. 40, no. 17, pp. 1034-1035, 2004.

[5] A. Tronquo, H. Rofier, C. Hertleer, and L. Van Langenhove, "Robust planar textile antenna for wireless body LANs operating in $2.45 \mathrm{GHz}$ ISM band," Electronics Letters, vol. 42, no. 3, pp. 142-143, 2004.
[6] G. Y. Chen, J. S. Sun, S. Y. Huang et al., "The Pliant Monopole Antenna (PMA) design for handset phone operation," in Proceedings of the 7th International Symposium on Antennas, Propagation and EM Theory (ISAPE '06), pp. 46-49, Guilin, China, October 2006.

[7] J. Jung, H. Lee, and Y. Lim, "Broadband flexible meander line antenna with vertical lines," Microwave and Optical Technology Letters, vol. 49, no. 8, pp. 1984-1987, 2007.

[8] J. Jung, H. Lee, and Y. Lim, "Broadband flexible comb-shaped monopole antenna," IET Microwaves, Antennas and Propagation, vol. 3, no. 2, pp. 325-332, 2009.

[9] D. E. Anagnostou, A. A. Gheethan, A. K. Amert, and K. W. Whites, "A direct-write printed antenna on paper-based organic substrate for flexible displays and WLAN applications," IEEE/OSA Journal of Display Technology, vol. 6, no. 11, pp. 558-564, 2010.

[10] H. K. Yoon, Y. J. Yoon, H. Kim, and C. H. Lee, "Flexible ultrawideband polarisation diversity antenna with band-notch function," IET Microwaves, Antennas and Propagation, vol. 5, no. 12, pp. 1463-1470, 2011.

[11] T. J. Jung, J. H. Kwon, and S. Lim, "Flexible zeroth-order resonant antenna independent of substrate deformation," Electronics Letters, vol. 46, no. 11, pp. 740-742, 2010.

[12] S. H. Choi, H. J. Lee, and K. B. Lee, "Flexible antenna based on composite right/left-handed transmission line," Electronics Letters, vol. 46, no. 17, pp. 1181-1182, 2010.

[13] J. Lee, S. I. Kwak, and S. Lim, "Wrist-wearable zeroth-order resonant antenna for wireless body area network applications," Electronics Letters, vol. 47, no. 7, pp. 431-433, 2011.

[14] W. C. Liu and C. F. Hsu, "Flexible CPW-fed double meandered monopole antenna for dual-band WLAN operation," Microwave and Optical Technology Letters, vol. 48, no. 8, pp. 1529-1532, 2006.

[15] M. L. Scarpello, D. Kurup, H. Rogier et al., "Design of an implantable slot dipole conformal flexible antenna for biomedical applications," IEEE Transactions on Antennas and Propagation, vol. 59, no. 10, pp. 3556-3564, 2011.

[16] S. R. Best, "A comparison of the performance properties of the Hilbert curve fractal and meander line monopole antennas," Microwave and Optical Technology Letters, vol. 35, no. 4, pp. 258-261, 2002.

[17] M. Z. Azad and M. Ali, "A miniaturized hilbert PIFA for dualband mobile wireless applications," IEEE Antennas and Wireless Propagation Letters, vol. 4, no. 1, pp. 59-62, 2005.

[18] J. Zhu, A. Hoorfar, and N. Engheta, "Bandwidth, cross-polarization, and feed-point characteristics of matched Hubert antennas," IEEE Antennas and Wireless Propagation Letters, vol. 2, pp. 2-5, 2003.

[19] J. Anguera, C. Puente, E. Martínez, and E. Rozan, “The fractal Hilbert monopole: a two-dimensional wire," Microwave and Optical Technology Letters, vol. 36, no. 2, pp. 102-104, 2003.

[20] D. Gala, J. Soler, C. Puente, C. Borja, and J. Anguera, "Miniature microstrip patch antenna loaded with a space-filling transmission line based on the fractal Hilbert curve," Microwave and Optical Technology Letters, vol. 38, no. 4, pp. 311312, 2003.

[21] J. Anguera, C. Puente, C. Borja, and J. Soler, "Fractal-shaped antennas: a review," Encyclopedia of RF and Microwave Engineering, vol. 2, pp. 1620-1635, 2005.

[22] J. Anguera, Fractal and broad-band techniques on miniature, multifrequency, and high-directivity microstrip patch antennas, 
Ph.D. thesis, Universitat Politècnica de Catalunya, Barcelona, Spain, 2003.

[23] S. S. Bor, T. C. Lu, J. C. Liu, and B. H. Zeng, "Fractal monopole-like antenna with series Hilbert-curves for WLAN dualband and circular polarization applications," Microwave and Optical Technology Letters, vol. 51, no. 4, pp. 876-880, 2009.

[24] D. O. Kim, C. Y. Kim, J. K. Park, and N. I. Jo, "Compact band notched ultra-wideband antenna using the Hilbert-curve slot," Microwave and Optical Technology Letters, vol. 53, no. 11, pp. 2642-2648, 2011.

[25] J. McVay, N. Engheta, and A. Hoorfar, "Numerical study and parameter estimation for double-negative metamaterials with Hilbert-curve inclusions," in Proceedings of the IEEE Antennas and Propagation Society International Symposium, vol. 3, pp. 328-331, July 2005.

[26] J. McVay, N. Engheta, and A. Hoorfar, "High impedance metamaterial surfaces using hilbert-curve inclusions," IEEE Microwave and Wireless Components Letters, vol. 14, no. 3, pp. 130-132, 2004.

[27] K. J. Vinoy, K. A. Jose, V. K. Varadan, and V. V. Varadan, "Resonant frequency of Hilbert curve fractal antennas," in Proceedings of the IEEE Antennas and Propagation Society International Symposium, pp. 648-651, July 2001.

[28] W. L. Stutzman and G. A. Thiele, Antennas Theory and Design, John Wiley \& Sons, New York, NY, USA, 2nd edition, 2003.

[29] J. C. Liu, B. H. Zeng, H. L. Chen, S. S. Bor, and D. C. Chang, "Compact fractal antenna with self-complementary hilbertcurves for WLAN dual-band and circular polarization applications," Microwave and Optical Technology Letters, vol. 52, no. 11, pp. 2535-2539, 2011. 

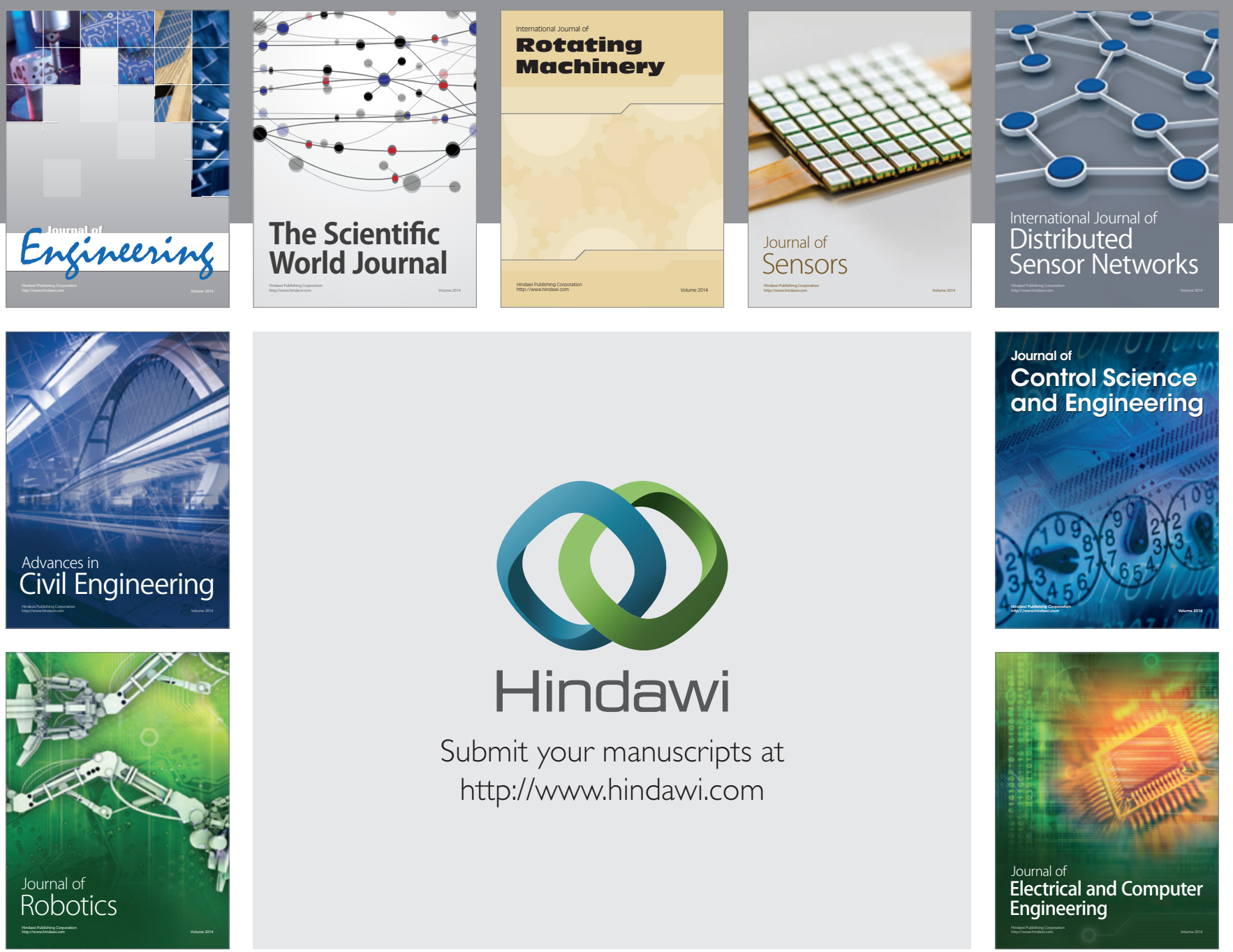

Submit your manuscripts at

http://www.hindawi.com
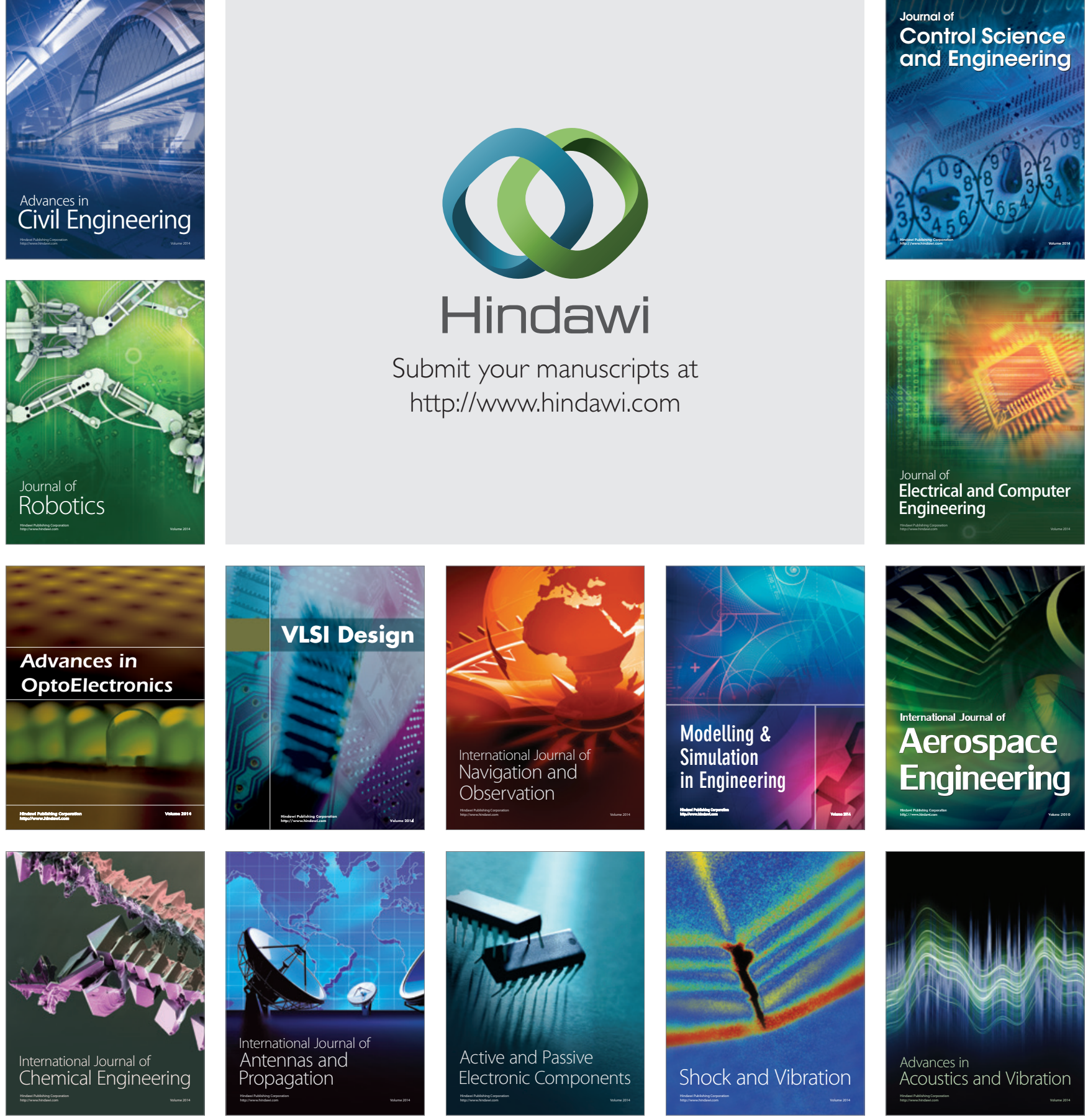